\title{
Univentricular Atrioventricular Connection. Review of the Current Therapeutical Possibilities
}

\author{
Edmar A tik
}

São Paulo, SP - Brazil

Unlike other congenital heart anomalies, univentricular atrioventricular connection is impossible to surgically repair most of the time.

Only certain types of univentricular atrioventricular connection can undergo surgical repair. These are cases where a $50 \%$ overriding of the right atrioventricular valve in the interventricular septum is present, which, from the anatomical point of view, characterizes the double inlet of a left single ventricle. From the physiological point of view, conditions exist for good development of the right ventricle through adequate flow from the right atrium. This development may favor the possibility of separation of the two circulations through interventricular septation.

This maneuver may be effective, even if the immediate postoperative evolution is somewhat unfavorable because the extension of the biological material used in the septation may reach up to $50 \%$ of the entire interventricular septum. Placement of such a large amount of material in the septum alters ventricular function and predisposes to interventricular residual defect.

Nevertheless, hemodynamic characteristics may be controlled by clinical and surgical measures providing a satisfactory long-term evolution.

On the other hand, most of the patients with univentricular atrioventricular connection undergo palliative surgeries depending on whether they have increased, normal, or decreased pulmonary blood flow.

In the first situation, increased pulmonary blood flow, pulmonary artery banding or the Damus-Kaye-Stansel operation is suggested. In decreased pulmonary blood flow, the 3 following surgeries are recommended: the Blalock-Taussig operation, bidirectional cavopulmonary anastomosis, and total cavopulmonary anastomosis. In normal pulmonary flow, either due to mild pulmonary stenosis or pulmonary artery hypertension with a balance in systemic and pulmonary flows, the watchful waiting approach is advised.

The main indication for surgery, either pulmonary

Instituto do Coração do Hospital das Clínicas - FMUSP

Mailing address: Edmar Atik - Incor - Av. Dr. Enéas C. Aguiar 44 - 05403-000 - São Paulo, SP - Brazil artery banding or the Damus-Kaye-Stansel operation, is the presence of congestive heart failure without hypoxemia with an oxygen saturation of approximately $90 \%$ and consequent mild cyanosis, and hematometry within the normal range.

The outcome of pulmonary artery banding is better in the absence of an abnormality in the left atrioventricular valve, either stenosis or even regurgitation, and also when the blood flow mixture is adequate at the atrial level due to the presence of an atrial septal defect.

Therefore, establishing communication between the two atria prior to surgery or even expanding an already existing atrial septal defect through balloon atrioseptostomy is recommended.

So reduction in the pulmonary venocapillary pressure prior to pulmonary artery banding makes this technique more effective in actually reducing pulmonary blood flow. Consequently, a greater restriction of the pulmonary flow is obtained, favoring a better immediate and late evolution. The lower pulmonary pressure protects pulmonary circulation as a whole, enabling the performance of the cavopulmonary type of functional correction in later years.

In regard to the indications for the Blalock-Taussig operation, the rules are the opposite and are determined by moderate to marked hypoxic findings with clear cyanosis and oxygen saturation lower than $80 \%$, hemoglobin higher than $15.5 \mathrm{~g} \%$, and hematocrit above $55 \%$. These rules also apply to partial or total cavopulmonary anastomoses. The age at operation, however, varies according to the type of surgery. The Blalock-Taussig operation is usually performed precociously before the $6^{\text {th }}$ month of life; above this age, partial cavopulmonary anastomosis is indicated, and above 2 years of age, total cavopulmonary anastomosis is recommended.

If patient does not meet the appropriate criteria for cavopulmonary anastomosis, the Blalock-Taussig technique may be considered and its use may be extended beyond 6 months of age.

The considerations about the greater effectiveness of pulmonary artery banding also apply to the BlalockTaussig operation. Therefore, previous atrioseptostomy is also necessary.

Corrective functional operations, such as the cavopulmonary type, become more effective if the criteria for 
their indication are present. Among them, the following are emphasized: mean pulmonary artery pressure $<15 \mathrm{mmHg}$; pulmonary vascular resistance $<2$ Wood units; adequate pulmonary arterial tree; absence of associated cardiac abnormalities, such as small ventricular septal defect or coarctation of the aorta, leading to valve regurgitation or myocardial hypertrophy, sinus rhythm; and preserved ventricular function.

Watchful waiting is recommended in more balanced situations, with similar pulmonary and systemic flows, such as in the presence of mild pulmonary stenosis and in pulmonary vascular disease, mainly in its initial phase, in which cyanosis is discreet, heart failure and cardiomegaly are absent, and a stable clinical condition exists.

In the first situation, presence of mild pulmonary stenosis, a more favorable opportunity for surgery should be awaited, if pulmonary blood flow progresively palls due to aggravation of pulmonary stenosis.

In the second situation, pulmonary vascular disease, cardiopulmonary transplantation is also considered.

At InCor (the Heart Institute of the medical school of the University of São Paulo), increasing surgical expertise has progressively reduced mortality with all types of surgery. We emphasize the importance of the appropriate choice of surgical technique based on the characteristics of each case.

Because of anatomical and functional similarities, univentricular atrioventricular connection and tricuspid atresia were grouped. From January '93 to January '99, the BlalockTaussig operation was performed in 123 patients (40 with univentricular atrioventricular connection and 83 with tricuspid atresia). Most of the patients were operated upon in the first year of life (70 patients [56.9\%] in the first 6 months, and 15 patients [ $12.1 \%$ ] between the $6^{\text {th }}$ and $12^{\text {th }}$ month of life). For the remaining patients, age distribution was as follows: 21 patients $(17 \%)$ between 1 and 5 years of age; 13 patients $(10.5 \%$ ) between 5 and 10 years of age; and 4 patients $(3.2 \%)$ between 10 and 32 years of age. Extension for the indication of the Blalock-Taussig operation to various ages, including adulthood, has been due to patients not meeting the already mentioned criteria for cavopulmonary anastomosis.

In regard to pulmonary artery banding, most of the 44 procedures were performed within the first year of life. Sixteen of these patients had tricuspid atresia and 28 had univentricular atrioventricular connection. Age distri- bution was as follows: 24 patients $(54.5 \%)$ were operated upon in their first 6 months of life; 12 patients $(27.2 \%)$ were operated upon between their $6^{\text {th }}$ and $12^{\text {th }}$ month of life; 6 patients $(13.6 \%)$ between 1 and 5 years of age; and 2 patients $(4.5 \%)$ between 5 and 10 years of age.

Regarding total cavopulmonary anastomosis, in the 36 patients operated upon ( 12 with univentricular atrioventricular connection and 24 patients with tricuspid atresia), a more homogeneous distribution in the various age groups occurred as follows: between 2 and 5 years of age, between 5 and 10 years of age, and between 10 and 19 years of age, corresponding to 15 patients $(41.6 \%), 13$ patients $(36.1 \%)$, and 8 patients $(22.2 \%)$, respectively.

The period for indication of bidirectional cavopulmonary anastomosis was extended to include the first year of life in $6(18.1 \%$ ) out of 33 patients (14 with univentricular atrioventricular connection and 19 with tricuspid atresia), and from 10 to 35 years of age in 2 patients (6\%). Most operations, however, were performed during the same periods of indication for total cavopulmonary anastomosis with 15 patients $(45.4 \%)$ between 1 and 5 years of age, and 10 patients $(30.3 \%)$ between 5 and 10 years of age.

Mortality rate for each of these operations was as follows: $8 \%$ for the Blalock-Taussig operation; $15 \%$ for the pulmonary artery banding; and 5\% for the total and partial cavopulmonary anastomoses.

This is an overview of our protocol for the most appropriate management of univentricular atrioventricular connection, which still challenges the best strategy.

Some doubts still exist in connection with particular cases of this anomaly. One example is the case of the small ventricular septal defect with or without coarctation of the aorta or interruption of the aortic arch in the neonatal period or in infancy. Should the Damus-Kaye-Stansel operation be performed in this case instead of pulmonary artery banding to reduce the number of operations until the desired cavopulmonary operation can be performed?

Furthermore, in similar cases with renal failure and an increase in creatinine and urea levels, one should operate upon or try to enhance the hemodynamic situation with the first stage operation?

Other situations may come to mind, but common sense is mandatory in determining the ideal stage for obtaining hemodynamic balance, as well as the balance of pulmonary and systemic blood flow. 